\title{
Perspectiva de los mercados laborales en un trabajo socialmente devaluado: el caso del trabajo sexual ${ }^{\star}$
}

\section{Perspective of the labor markets \\ in a socially devalued work: \\ the case of sex work}

Carlos Alfonso Laverde Rodríguez**

Recibido: 18 de octubre de 2016

Revisado: 30 de noviembre de 2016

Aprobado: 18 de enero de 2017

\section{Resumen}

A partir de una noción ampliada de los mercados de trabajo, se plantea que en el trabajo sexual existe un mercado laboral el cual, al igual que otros mercados, está sujeto a regulaciones extraeconómicas. Desde esta perspectiva, se sostiene que, además de

* Artículo de investigación producto de la tesis en desarrollo titulada Apropiación subjetiva de derechos laborales: relatos de vida de mujeres trabajadoras sexuales organizadas en defensa de sus derechos en la ciudad de Bogotá, para optar al título de doctor en Ciencia Social con Especialidad en Sociología, por El Colegio de México.

** Candidato a Doctor en Ciencia Social con Especialidad en Sociología por El Colegio de México. Correo electrónico claverde@colmex.mx 
regulaciones jurídicas e institucionales, existen formas de control socialmente construidas, que para el trabajo sexual se fundan en un proceso de estigmatización. Se propone ampliar la idea de regulación a partir de procesos sociales más complejos, como el descrédito de la actividad, que se traduce en un control efectivo de quienes realizan este trabajo. Se hace uso de los conceptos "trabajo no clásico", a partir de la propuesta de De La Garza (2010), y "capital social”, desde la perspectiva de Portes (1999), para analizar las formas de control y las disputas por el reconocimiento de significados y prácticas que están presentes en el trabajo sexual.

Palabras clave: Mercado de trabajo, trabajo sexual, estigmatización, capital social.

Clasificación JEL: J40, J46, J7 1, J24

\section{Abstract}

From an expanded notion of labor markets, the sex trade is taken as a job market, which, like other markets, is subject to extra economic regulations. From this perspective it is supported that besides legal and institutional regulations, there are socially constructed forms of control, which for the sex work are founded in a process of stigmatization. It is proposed to expand the idea of regulation from more complex social processes as the discredit of the activity that is translated to an effective control of the ones who carry out this work. The concept of the non-classic work taken from the proposal of De La Garza (2010) and of the social capital, from the perspective of Portes (1999), are used to analyze the forms of control and the disputes for the recognition of meanings and practices that are present in the development of the sex work.

Keywords: Labor market, sex work, stigmatization, social capital.

Classification JEL: J40, J46, J7 1, J24 


\section{Introducción}

Los mercados de trabajo tienen diferentes mecanismos de regulación. Esta premisa se ha planteado ampliamente en todo el desarrollo de la teoría económica, especialmente, desde una tendencia neoclásica que proclama la autorregulación de los mercados.

Sin embargo, desde otra orilla, la sociología ha retomado la idea de la regulación desde factores extraeconómicos que se orientan a normas y reglas institucionalmente atribuidas y socialmente construidas e internalizadas. Esta idea permite esbozar, de forma muy general y básica, la intención de una sociología del trabajo tal como la proponen Kalleberg y Sorensen (1979), Maruani (2000), Araújo (2009) y De La Garza (2010), entre otros.

Con fundamento en la propuesta de la sociología de los mercados de trabajo, planteo que el comercio sexual es un mercado laboral el cual, al igual que otros mercados laborales, está sujeto a regulaciones extraeconómicas. Así mismo, considero que el comercio sexual está sujeto a especificaciones particulares que lo constituyen como un trabajo.

En este sentido, desde un marco laboral, además de regulaciones jurídicas e institucionales existen cánones que se construyen socialmente, y que en el caso del trabajo sexual están mediados por un proceso de estigmatización en el que se disputa o se juega una lucha por el reconocimiento.

Retomar el concepto de trabajo en el comercio sexual no implica dejar de reconocer formas en las que se vulneran los derechos, como la trata de personas y la explotación sexual. Por lo tanto, me refiero a trabajo sexual como la prestación de servicios sexuales remunerados a cambio de una retribución económica y que se realiza de forma voluntaria y consensuada.

Como objetivo de este artículo intento responder el siguiente interrogante: ¿cómo se construyen elementos de regulación en el interior del trabajo sexual? Para responder a esta pregunta, retomo la noción de mercado de trabajo no clásico desarrollada por De La Garza (2010) y los elementos de regulación que se proponen desde las teorías del capital social, vistos en la perspectiva de Portes (1999), nociones que reconstruyo a partir del caso de trabajadoras sexuales del barrio Santafé, en la ciudad de Bogotá. Lo particular de este caso es que desde el año 2001 se viene adelantando un proceso de reglamentación orientado hacia la regulación y defensa de los derechos laborales. Este proceso se lleva a cabo a partir de una sentencia de la Corte Constitucional colombiana (Sentencia T/629 de 2010) que enfatiza en la necesidad de que los legisladores retomen la noción de trabajo como concepto que reivindica los derechos de esta población. Por su parte, la Sentencia T/594 de 2016 apunta y refuerza lo expuesto en la Sentencia T/629 de 2010 
Como hipótesis considero que el mercado del trabajo sexual, si bien puede tener regulaciones que operen bajo la lógica del mercado, requiere construir la idea de regulación a partir de procesos más complejos, como el de estigmatización, que permite un control efectivo con la menor inversión de esfuerzos y recursos por parte de quienes se pueden usufructuar de forma indirecta de esta actividad económica.

Con la intención de responder a la pregunta general, abordaré el presente texto en el siguiente orden. En primer lugar, para posicionar el trabajo sexual dentro de una lógica laboral, retomaré la discusión de la construcción social de los mercados de trabajo. En un segundo momento, con base en De La Garza (2010), desarrollaré la propuesta de trabajo no clásico, concepto que sirve como bisagra entre el estudio de los mercados de trabajo y el trabajo sexual como una forma particular de mercado. Posteriormente, una vez posicionado el trabajo sexual como una forma de trabajo (no clásico), plantearé, desde la visión de Portes (1999), elementos sobre la forma en que el capital social que se construye en este mercado impone formas de regulación formal, pero también no formal.

Finalmente, propongo que la regulación social en la que está implícita la lucha por el reconocimiento de este mercado laboral está sujeta a un proceso de estigmatización que se manifiesta en una lucha por los significados y las prácticas presentes en el trabajo sexual.

\section{Construcción social del mercado en el comercio sexual}

Maruani (2000) exhorta al estudio del trabajo desde una perspectiva sociológica que permite abordarlo como una construcción que persigue una forma de racionalidad basada en normas y reglas. Lo social se centra en el análisis del trabajo, y no exclusivamente en los mecanismos económicos.

En el mismo sentido lo plantean Kalleberg y Sorensen (1979), quienes definen el mercado de trabajo de forma abstracta, como el lugar en el que los trabajadores cambian su fuerza de trabajo por salarios, estatus y otras recompensas. El concepto, por lo tanto, se refiere ampliamente a las instituciones y prácticas que rigen la compra, la venta y el precio de los servicios laborales.

La concepción que proponen Kalleberg y Sorensen (1979) discute frontalmente la noción clásica de mercado de trabajo que tradicionalmente ha impuesto la economía desde su perspectiva neoclásica. Es necesario recordar que, desde la perspectiva neoclásica, el mercado de trabajo se fundamenta en la libre competencia y que, en tal virtud, el equilibrio se alcanza cuando el oferente u empleador maximiza su utilidad en el nivel de absorción de mano suficiente para que su ingreso marginal sea igual al costo marginal. En este estado, en el punto óptimo se logra vaciar el mercado o por lo menos se alcanza 
la absorción de la oferta de mano de obra disponible, dejando una proporción que se denomina tasa natural de desempleo, la cual se ajusta con la propia dinámica del mercado, conocida también como desempleo friccional (Carrasco, Castaño y Pardo, 2011).

Según Kalleberg y Sorensen (1979), la economía neoclásica concibe que existe una regulación autónoma del mercado, sin embargo, olvida la interacción con fuerzas sociales y políticas que transforman las relaciones entre empleados y empleadores, así como todo el sistema productivo.

Esta transformación entre actores involucrados en el sistema productivo define incluso el estatuto propio del trabajo. Por mencionar un ejemplo, desde una perspectiva de género, en algunos casos se valora, se define y se otorga reconocimiento social a un trabajo y su salario, así como a otros no se les atribuye este mismo estatuto, diferenciado a partir de una construcción cultural que ha otorgado roles productivos; por tal razón, existen ciertos trabajos considerados como "masculinos" y otros como "femeninos", segmentados por su desigual ponderación en el mercado de trabajo, ya que, como lo afirma Téllez (2001, p. 5): "Las representaciones ideológicas influyen en la forma en que las personas se integran en el mercado de trabajo modelando sus preferencias".

Tal es el caso de trabajos como el doméstico, el cual está subestimado como actividad secundaria que se remite a las tareas de cuidado atribuidas a un rol más o menos naturalizado de las mujeres. Tal como lo afirman Tilly y Tilly (1998), el valor social del trabajo y la forma de concebirlo se insertan en relaciones de poder que dan cuenta de la desigualdad social, en este caso, por razón del género.

En esta misma vía, el trabajo sexual no goza del mismo reconocimiento que otro trabajo. La muestra más clara de que esto ocurre es el marco jurídico, en el que no se asume este trabajo como tal, por lo tanto, no se reconocen una serie de derechos que en materia jurídica deberían estar amparados, por ejemplo, el derecho a seguridad social, salud, pensiones, entre otros.

Desde una perspectiva de género, el trabajo sexual implica una ruptura con roles atribuidos socialmente a las mujeres, tales como una sexualidad ejercida preferentemente en el marco del matrimonio, la fidelidad y la maternidad. A este trabajo se le atribuye una identidad desacreditada (no solo por romper con los cánones anteriores, sino por cobrar o recibir dinero a cambio) y que, por ende, se extiende a las mujeres que lo ejercen.

Tal como lo proponen Tilly y Tilly (1998), las lógicas inscritas en el mercado están basadas en relaciones sociales en las que se reproducen relaciones de poder. Estas lógicas suponen que algo está en juego y que en la mayoría de casos está en disputa el reconocimiento, ya sea de salario, jerarquía, derechos y legitimidad. 
Ahora bien, lo que se ha planteado es una noción de mercado laboral abstracta y generalizable, sin embargo, es necesario señalar la importancia de la ubicación de estas nociones. La noción de un mercado como relación social que se construye en el espacio y que, por consiguiente, responde a sus dinámicas, es elaborada por Peck (1996), quien considera que, según la teoría ortodoxa de la economía, el mercado laboral local se proyecta directamente en un espacio. Cada mercado laboral local funciona de acuerdo con una lógica universal. En esta perspectiva, el espacio es pasivo y meramente contextual, y el mercado laboral local solamente es como un contenedor para procesos universales, pues las leyes del mercado existen más allá de las contingencias de la historia y la geografia. Así, estos operan independientemente de lo que ocurra en el contexto y las leyes tienen los mismos efectos en cada lugar.

Sin embargo, según Peck (1996), no hay justificación teórica para que la situación geográfica se considere como una "contingencia" en el nivel nacional. Si la estructura y los procesos del mercado laboral varían entre naciones, también deben variar en espacios locales; de igual forma, si sus procesos están formados por un contexto, entonces se pueden asociar con variaciones espaciales.

El lugar donde se desarrolla el comercio sexual depende de un marco jurídico que organiza las formas de interacción, ya sea hacia un plano de prohibicionismo, de abolición o de reglamentación. Esto resulta relevante debido a que lo jurídico define las relaciones sociales en cuanto a prácticas legales o ilegales y que tienen implicaciones si se consideran legítimas o ilegitimas.

Por citar un ejemplo, en el caso de la ciudad de Bogotá, en $2001^{1}$ surgió un proceso reglamentario para localizar y definir zonas en las que se puede ejercer el trabajo sexual bajo la mirada institucional. Así, en los lugares donde se construyó el comercio sexual históricamente - a partir del momento de la emisión de un marco reglamentario que tendía además hacia la protección de los derechos de las trabajadoras y los trabajadores sexuales-, se abrió un proceso de legitimación que ha tenido efectos en la apropiación de derechos, que en otro modelo jurídico no sería tan claro.

Por lo tanto, el planteamiento de los mercados situados es útil en mercados laborales como el trabajo sexual, a los que no se puede extender una explicación global de fenómenos que se sitúan en relaciones políticas, históricas, culturales y, además, en el marco de un contexto jurídico. Cada mercado laboral tiene una especificidad que da cuenta de relaciones y permite entender cómo se configuran las relaciones de poder.

Ahora bien, con el fin de posicionar conceptualmente el trabajo sexual a la luz de relaciones de poder ancladas en una lógica de mercado, desarrollaré en el siguiente apartado el concepto de trabajo no clásico propuesto por De La Garza (2010), con el fin de

1 Ver Decreto 400 de 2001. 
ampliar en la naturaleza de este trabajo sus características y dimensiones, en relación no solamente con quienes desarrollan este trabajo, sino con los actores involucrados en el comercio sexual.

\section{Trabajo sexual como trabajo no clásico}

Desde una perspectiva sociológica, el trabajo es una forma de interacción entre personas que intercambian objetos materiales y simbólicos. De La Garza (2010) afirma que, finalmente, todo trabajo es una construcción y un intercambio de significados. Este primer planteamiento tiene importantes consecuencias en el análisis sociológico de los mercados de trabajo. Un intercambio supone una forma de relación social en la que intervienen los interesados en algo que se mercantiliza, algo que resulta de interés y, por lo tanto, refleja un proceso de construcción sobre los valores que están en juego en el proceso de negociación. Como relación social se puede entender igualmente la puesta en escena de significados y símbolos que se escapan de la atención misma del objeto de intercambio, es decir, del trabajo que se cambia por un salario. Hay elementos suficientes para establecer un orden en la interacción, en la que se busca ya sea posición social, estatus, jerarquías. En otras palabras, se traduce como una relación de poder en la que se juegan los intereses de quienes forman la relación laboral.

Desde esta perspectiva, el trabajo -entendido como parte de un proceso social más amplio- requiere de un análisis que complejice la lógica de la interacción. De La Garza (2010), en su texto Hacia un concepto ampliado de trabajo, propone tres ejes de desarrollo conceptual: 1) proceso ampliado de trabajo, 2) proceso ampliado de regulación y 3) lógica de la ocupación. Para el presente texto propongo retomar los dos primeros ejes en relación con el trabajo sexual.

En un primer momento, De La Garza (2010) señala el proceso ampliado de trabajo, que involucra la presencia de actores adicionales a la concepción tradicional capital/trabajo, es decir, todas las personas que se involucran para que un trabajo sea llevado a cabo, independiente de si se es asalariado o no.

En el comercio sexual, los actores involucrados en el proceso de trabajo son principalmente siete: 1) clientes; 2) administradores; 3) trabajadores (de establecimientos y calle); 4) otras trabajadoras y otros trabajadores sexuales; 5) autoridades (principalmente policía); 6) asociaciones y organizaciones civiles (asociaciones de vecinos, ONG); y 7) comerciantes del sector.

El papel de los clientes resulta crucial en este trabajo, ya que estos son el objeto mismo de la oferta del trabajo sexual. En contraste con otros trabajos, la cercanía e intimidad que se establece con el cliente en el trabajo sexual no se limita a la prestación de 
un servicio sexual. De acuerdo con la Secretaría Distrital de Integración Social (SDIS, 2009), en Bogotá, los datos muestran que la relación sexual entre trabajadora sexual y cliente puede durar entre 10 minutos y media hora, sin embargo, el tiempo en el que pueden estar los clientes es más extenso. Esto lo que demuestra es que hay otro tipo de relaciones que van más allá de la prestación del servicio sexual.

En este sentido, el estudio manifiesta que el $23 \%$ de los hombres afirmó buscar siempre a la misma mujer con quien ha tenido relaciones anteriormente, lo cual habla de un trabajo que se relaciona con lo afectivo y en el que se tejen relaciones de cercanía que van más allá de una simple negociación de un servicioº

Nosotras somos un aliciente para ellos, ellos siempre llegan con una tristeza, con un aburrimiento, y nosotras estamos con una sonrisa, con una pierna así toda chévere, toda así, y el hombre se va a sentir, aunque el hombre llegue con su acongojo, con su problema, su dilema, siempre va llegar a un sitio de esos y va a encontrar a alguien con una sonrisa y con una palabra que lo va a dejar [...] (Laverde, 2014).

En estos términos, el cliente no realiza una transacción con la trabajadora sexual, sino que además crea vínculos afectivos que se presentan en el orden de la interacción. Esta característica rompe por lo menos con la idea de una lógica comercial en la que el trabajo implica estrictamente la producción de un bien o servicio. Para la producción del servicio se requiere de la participación activa del cliente para ejecutar el servicio que se solicita.

Ahora bien, los administradores y los trabajadores de los complejos de prestación de servicios son fundamentales para el desarrollo del trabajo sexual, pero también para la forma en que las mujeres experimentan y afrontan el trabajo. La función principal de estos actores es brindar la seguridad y las condiciones propicias para clientes y trabajadoras dentro de los establecimientos.

Otro grupo actor lo conforman las compañeras trabajadoras, quienes pueden ser una fuente de protección ante los peligros de su trabajo, pero también pueden ser una amenaza. La disputa por los clientes puede ser un motivo para que el ambiente en los lugares de trabajo sea tenso y en ocasiones pueda tornarse violento.

Sumadas a los anteriores actores, están las autoridades, que juegan un papel preponderante en el escenario del trabajo sexual. En Bogotá, a partir de la reglamentación de 2001, el trabajo sexual en sí mismo no es ilegal, por lo que las autoridades no pueden ejercer alguna sanción a su trabajo. Sin embargo, algunas autoridades, como la policía,

2 En este sentido, considero que el trabajo sexual es una de las formas del trabajo no clásico, concepto propuesto por De La Garza (2010), quien afirma que la preferencia del consumidor (cliente) no solo se basa en un precio, sino en la producción social de la ocupación, que en este caso vincula aspectos afectivos. 
siguen siendo actores que son definidos como vulneradores de los derechos de estas mujeres.

También está el grupo actor directo compuesto por las organizaciones que buscan defender los derechos de la población. Pero también existen organizaciones como la de los vecinos que están en contra de dicha actividad u otras que tienen su propia agenda, ya sea para defender los derechos laborales o apoyar la abolición de dicha actividad.

Finalmente, están los comerciantes que, si bien no se relacionan de forma directa con la organización del trabajo sexual, sí apoyan de forma indirecta todo el proceso, como la prestación de servicios de peluquería, restaurante, boutiques, entre otros, dinámica que es particular en este medio, ya que incluso los precios se establecen de acuerdo con la dinámica de la zona ${ }^{3}$.

En este orden de ideas, los actores que se relacionan en el proceso de trabajo tienen una función de control diferente en comparación con la tradicional relación laboral. En la clásica concepción de la forma de trabajo, tal como la considera De La Garza (2010), el control supone tiempos de trabajo, momentos de intervención, ritmos y productividad que están dirigidos desde una posición superior frente a los trabajadores. Sin embargo, desde la perspectiva de los servicios, como lo es el trabajo sexual, el cliente participa o está implicado en el proceso de producción y desplaza la tradicional forma de control industrial, en la que solo se contempla al empleado y al empleador.

En el tipo de servicios que se prestan en el comercio sexual no se produce para almacenar, como lo sería la producción de cualquier bien, sino que se consume en el mismo momento de la producción. En esta vía, las relaciones sociales en el proceso de trabajo son fundamentales, pues están implícitas en el proceso de producción mismo, ya que se vincula al consumidor para la producción del servicio.

En el comercio sexual, el cliente está inmerso en el proceso de producción en dos sentidos: por una parte, en la prestación del servicio sexual, y, por otro lado, en un proceso de creación simbólica y afectiva que recrea la producción de intimidad. Es un trabajo de simulación y producción simbólica. En términos de De La Garza (2010), en este tipo de actividades de producción inmaterial, el producto no está separado del propio momento de la producción, de manera que las fases económicas tradicionales se comprimen en un solo acto.

3 De acuerdo con el trabajo de campo desarrollado en enero de 2016, he podido observar que los bienes y servicios requeridos por trabajadoras sexuales en el sector del barrio Santafé pueden subir de costo debido a la alta demanda, por una parte, pero también a que, en muchas ocasiones, algunas de las trabajadoras sexuales reciben de forma continua sumas importantes de dinero y esto es aprovechado por las dinámicas del comercio, por ejemplo, el incremento de precios en bienes como ropa que es usada en los establecimientos o servicios como peluquerías, comida, etc. 
Por ende, en el proceso de producción tal como se ha planteado, resulta de interés fundamental comprender cómo se construye el proceso de regulación del trabajo. Si, por una parte, en el comercio sexual, la producción se realiza con la participación directa e inmediata del consumidor, resulta necesario entender cómo se establecen el proceso de control y la regulación en el encuentro con el consumidor. En otras palabras, resulta necesario conocer cómo se construye la relación con los actores directos y cómo se regulan los procesos de trabajo en el comercio sexual. En términos de Portes (1999), interesa saber cuáles son los capitales que están en disputa en el caso del trabajo sexual.

\section{Capital social y formas de regulación}

El concepto de capital social ha sido retomado desde múltiples perspectivas, por lo tanto, ha tendido a diversificarse, llegando incluso a correr el riesgo de perder su valor heurístico. Sin embargo, si algo parecen haber tenido en común las corrientes teóricas que han trabajado el concepto, en especial desde la sociología y la economía, es la concepción positiva que se ha atribuido a este.

Como crítica a estas perspectivas, Portes (1999) ha resaltado el rol, que no siempre puede ser positivo, del capital social. Es posible identificar cuatro consecuencias negativas: 1) exclusión social a los extraños que componen una red; 2) la fuerte integración que se deriva del capital social en las comunidades puede crear una sobrecarga de trabajo a sus integrantes; 3) como consecuencia del punto anterior es posible que existan restricciones a la libertad individual; y 4) normas niveladoras hacia abajo, que implican un estancamiento de los integrantes sobresalientes dentro de una red social carente de capital social.

Esta perspectiva, que sostiene los efectos positivos, también tiene efectos perversos que incentivan mayores niveles de exclusión y desigual social, por lo tanto, resulta un enfoque apropiado para analizar un mercado laboral como el caso del trabajo sexual, el cual tiene fuertes connotaciones negativas por cuenta de las representaciones sociales sobre un trabajo desacreditado socialmente.

Al respecto, Portes (1999) ha señalado tres usos que se han hecho tradicionalmente sobre el concepto de capital social: como fuente de control social, como fuente de apoyo familiar y como fuente de beneficios por medio de las redes extrafamiliares. Para efectos del mercado del sexo, considero pertinente señalar el uso del concepto del capital social desde la óptica del control social.

Retomo el capital social de acuerdo con la perspectiva de Bourdieu, citado por Portes (1999), quien considera que este tipo de capital es "el agregado de los recursos reales o potenciales que se vinculan con la posesión de una red duradera de relaciones más o 
menos institucionalizadas de conocimiento o reconocimiento mutuo" (p. 248). La disputa por el conocimiento y el reconocimiento dentro de una red es precisamente la que se pone en juego en una actividad desacreditada como el trabajo sexual. Por lo tanto, el capital social funciona como una serie de reglas, algunas en el plano de lo jurídico, pero, además, como una serie de orientaciones normativas extralegales que dan cuenta del control social de una actividad.

El capital social produce una serie de lazos sociales que generan marcos de valoraciones y significados. Así, es atribuido cierto tiempo de comportamientos que deben corresponder entre los integrantes de la red que lo conforman, ya que existe la obligación de reciprocidad, como pagar las deudas a tiempo o tener cierto tipo de comportamiento ciudadano, por ejemplo, no arrojar basura. En este marco de interiorización de normas, el capital social resulta un instrumento analítico para comprender los procesos de regulación en el interior del comercio sexual. No solo son esperables conductas que se circunscriban al ámbito del marco jurídico, sino que además en las interacciones sociales, bajo la lógica de este mercado, son esperables conductas hacia los clientes, administradores, otras y otros compañeros. Adicionalmente, el capital social permite entender la forma en que se acumulan conocimientos colectivos en un proceso de interiorización de normas de acuerdo con el contexto, que se traduce en una forma de adaptación a este mercado laboral.

Murphy y Venkatesh (2011) realizaron una investigación con mujeres trabajadoras sexuales en la ciudad de Nueva York y lograron identificar particularidades que les permitieron definir que quienes realizan esta actividad económica desarrollan prácticas profesionalizantes y carreristas por ser para ellas un modelo de generación de empleo e ingresos.

En esta misma vía, Rivers-Moore (2010) ha señalado, a partir de su investigación en Costa Rica, que el trabajo sexual no es realizado solamente como una forma de sobrevivir, sino que, por el contrario, en el trabajo sexual existe una lógica de mercado que es aprovechada por las trabajadoras sexuales como estrategia para poder mantener un alto nivel de recursos que en otro empleo no lograrían obtener.

Por otra parte, Ding y Ho (2013) dieron un paso más adelante al proponer que en el trabajo sexual se desarrolla un tipo de capital denominado capital sexual ${ }^{4}$, el cual puede ser entendido como una expresión de la acumulación de conocimiento desde el momento

4 Este mismo concepto puede ampliarse en la propuesta de Hakim (2011), quien, a partir de la idea de capitales de Bourdieu, construye la noción de capital erótico, que se plantea desde la diferenciación de género y puede servir como fuente de explicación de lo erótico como un capital transferible en otros capitales, como el económico y/o cultural. En este momento retomo solamente el concepto de capital sexual que me permite hacer explícito el carácter sexual explícito que no es tan claro en lo erótico. 
en el que se ingresa al trabajo sexual, así como en escenarios previos en los que se valora la sexualidad femenina como un mecanismo para alcanzar objetivos específicos.

Ahora bien, en el trabajo sexual, y desde una perspectiva de regulación, el capital social se genera gracias a lo que Berger y Luckman (1968) llamarán internalización, concepto que alude a la adecuación que tiene un colectivo ante la aplicación de categorías. Es decir, la internalización se relaciona con un proceso de socialización secundaria que se forma a partir de un contexto de trabajo que tiene connotaciones tanto sociales como jurídicas. Las trabajadoras sexuales internalizan un orden social que, si bien puede estar marcado por un pretendido acceso a derechos laborales que puede o no cumplirse, también en él está presente una lógica del mercado laboral que reproducen para lograr sus objetivos.

Como se ha señalado, el capital social es producto de recursos que se ponen en juego en una red duradera de relaciones. Este capital crea procesos de regulación y, por lo tanto, de comportamientos esperados. Además, se ha mencionado que la particularidad de este trabajo es la atribución de características sociales que lo desacreditan, por tal razón, sus mecanismos de regulación operan de una forma diferente a otros mercados laborales. Por consiguiente, considero que, en el caso del trabajo sexual, la disputa o los intereses que se ponen en juego desde una perspectiva del capital social se dan por el reconocimiento de derechos laborales en un contexto de estigmatización.

En el siguiente apartado plantearé finalmente las coordenadas de un debate más amplio y que permita dar pistas para resolver los siguientes interrogantes: ¿cómo se regula este trabajo en sus formas extraeconómicas? ¿Bajo qué lógicas sociales se inserta la regulación del trabajo sexual? Planteo un marco conceptual desde el cual aproximarse a estas respuestas, siguiendo la noción de estigmatización de Goffman (1998) y de desarrollos posteriores, como los de Parker y Aggleton (2002).

\section{La estigmatización como proceso de regulación}

La estigmatización es un proceso social que en el caso del trabajo sexual opera como una forma de regulación de este mercado laboral. Sin embargo, como proceso social no está exento de un continuo enfrentamiento ni de la lucha por el reconocimiento. Esto implica que las trabajadoras sexuales no son pasivas ante una marca atribuida por cuenta de su trabajo desacreditado, sino que desde las representaciones sociales atribuidas se reconfiguran y experimentan las valoraciones y los significados.

En el trabajo sexual existe un proceso de estigmatización que se vive en dos contextos: el primer escenario alude a un contexto social más amplio que no está directamente relacionado con la actividad, pero que tiene representaciones acerca del comercio sexual; 
el segundo está compuesto por las relaciones que se establecen con los agentes directos involucrados en el mercado laboral.

En el primer escenario, los significados sociales respecto al trabajo sexual se erigen con fundamento en las nociones culturales que históricamente han construido una imagen devaluada de la trabajadora sexual: la prostituta. Instituciones como la familia, el Estado y la religión han estructurado la devaluación de la identidad de la trabajadora sexual en función de la transgresión a las concepciones sobre la sexualidad y el género. El conjunto de la sociedad espera que la comunidad estigmatizada acepte la identidad asignada a su grupo y que incorpore la visión del resto de la sociedad, que se acepte en su posición y que la recuerde para no considerarse nunca tan igual como los demás.

En un segundo escenario, la estigmatización como proceso social se da en el marco de las interacciones sociales dentro de la comunidad estigmatizada y los actores cercanamente relacionados, otorgando otro sentido al orden de la interacción. En este sentido, la lógica del comercio sexual implica, además de una transacción comercial, todo un conjunto de construcción e interacción de significados que dan otro significado a la estigmatización.

Es decir, la estigmatización no solo funciona como un atributo impuesto a una comunidad o individuos, sino que se interpreta y reinterpreta por quienes son estigmatizados. Por citar un ejemplo, en un grupo de discusión con mujeres trabajadoras sexuales en Bogotá, una de ellas señaló:

[...] yo al menos pongo el culo y cobro y no me arrastro y lo doy gratis, -así se lo dije- $[\ldots]$ mi hermana sabe que yo soy más puta que un verraco (Laverde, 2014).

En este caso, la mujer asume el estigma con una carga valorativa invertida frente a la mujer que la cuestiona, pues valora de forma positiva el uso que hace de su sexualidad en términos económicos, en oposición a su interlocutora, que lo hace "gratis". Esta mujer reafirma su posición social y valora de manera positiva su actividad, que tiene retribución económica; mientras que la otra mujer, que en el contexto de la frase se refiere a una mujer casada, presta servicios sexuales a su esposo, pero de forma gratuita, lo que, de acuerdo con el argumento expuesto, resulta poco satisfactorio y cuestionado.

La reinterpretación del proceso de estigmatización evidencia un proceso de regulación. Por estar inserta en relaciones de poder, la regulación de este mercado laboral - que en el trabajo sexual se orienta en un proceso de estigmatización- implica negociaciones, resistencias y/o aceptación que no son unidireccionales, sino que suponen un proceso social de continua reconstrucción.

En este sentido, Shih (2004) ha investigado los procesos exitosos en los que, a partir de un estigma, se consolida una identidad que utiliza su condición para superar efectos negativos. Quien es estigmatizado concibe su condición asignada socialmente como una 
situación que permite posibilidades de empoderamiento. Según la autora, el efecto del estigma se puede revertir y posibilitar en tres procesos positivos: 1) una posible compensación por efectos del estigma, ya que las personas que lo poseen se esforzarán más por ser asertivos y alcanzar sus metas con base en su condición social; 2) interpretación estratégica del entorno, que se asume al comparar sus acciones dentro de un marco desfavorecido en contraste con grupos que no poseen el estigma, por lo que pueden sentir que sus avances son más valorados por la dificultad que supone la desigualdad desde la que se sitúan los grupos estigmatizados; y 3 ) las personas que tienen una identidad caracterizada por el estigma pueden recurrir a múltiples identidades en las que se protegen de los efectos negativos del estigma o pueden apelar a una de ellas en su defensa , así tienen más oportunidades de apoyo social. Según Shih (2004), los individuos pueden enfatizar estratégicamente las identidades que son valoradas y restar importancia a las que no lo están, de acuerdo con el contexto social en el que se desenvuelven.

El proceso de estigmatización en el trabajo sexual es asimismo usado de manera estratégica desde la misma población en la cual recae el descrédito. Por una parte, están quienes desde la posición del trabajo sexual reclaman la normalización y el acceso a derechos laborales desde el plano jurídico y social, luchando por su reconocimiento y rechazando, por lo tanto, el estigma asociado con su actividad; por otra parte, están quienes rechazan y luchan contra la permanencia en esta actividad y reclaman ante la sociedad y el Estado soluciones para encontrar una fuente de ingresos que les resulte digna y que las aleje de esta actividad por cuanto no la consideran, desde ningún punto de vista, como un trabajo.

De esta forma lo que se intenta plantear es que, si bien el estigma - entendido como una etiqueta negativa o como sanción ante la transgresión de la norma- también se puede revertir en un punto de partida para acciones colectivas - sin importar el destino y los objetivos de la lucha, y no únicamente como un proceso en una sola vía que--, si bien atenta contra la población en la que recae, parece no darles visibilidad como actores sociales.

\section{Conclusiones}

Los mercados laborales se regulan y responden no solo a reglas y dinámicas económicas, sino que además responden a contextos que desbordan lo eminentemente económico. Los mercados laborales forman parte de construcciones sociales, por lo tanto, las formas de regulación se sustentan en arreglos sociales y, por consiguiente, no son estables por principio ni responden a modelos teóricos que se puedan predecir, como se acostumbra hacer en la teoría económica más ortodoxa. 
A la luz de la complejidad de los procesos sociales, el comercio sexual y específicamente el trabajo sexual tienen características que los ubican dentro del campo de estudio de los mercados laborales, ya que persiguen una determinada forma de racionalidad que se fundamenta en normas y reglas para su funcionamiento.

En este mercado resultan interesantes las diferentes formas de racionalidad a las cuales responde y sus lógicas de funcionamiento, que están influidas por representaciones sociales atribuidas a este trabajo. Las trabajadoras sexuales prestan servicios sexuales remunerados, lo que resulta contradictorio con roles atribuidos desde una perspectiva androcéntrica a las mujeres.

Debido a la especificidad que encierra este mercado, se distancia de las clásicas definiciones de trabajo, por tal motivo, es necesario posicionar el trabajo sexual como una forma no clásica de trabajo. Esto significa que el trabajo sexual no responde a las dinámicas clásicas de capital-trabajo ni a la relación con determinados medios de producción que generan un producto comercializable.

Retomando el concepto ampliado de trabajo que propone De La Garza (2010), el trabajo sexual es una clase de trabajo en el que no se pueden almacenar servicios, por ende, el consumo se realiza en el mismo momento de su producción. Esta forma de producción involucra de forma directa en el proceso al mismo consumidor en una relación cara a cara en la que aspectos como la afectividad juegan un papel central.

Por lo tanto, estos procesos subjetivos que están contenidos en el trabajo sexual aluden a relaciones sociales en las que se juegan intereses y procesos sociales más amplios que pueden estar referidos a las nociones sobre las mujeres y las concepciones de género. Estas nociones se entretejen como formas latentes de regulación del trabajo sexual y se manifiestan en la estigmatización, que funge como principio de regulación en este trabajo.

Lo que resulta de especial interés es que el proceso de estigmatización no se experimenta en este mercado como un proceso unidireccional, sino que puede manifestarse por medio de negociaciones en las cuales el capital social juega un rol central.

Desde la perspectiva del capital social que propone Bourdieu -retomado por Portes (1999)-, las redes sociales en las que se construye este capital se orientan hacia una institucionalización de relaciones grupales que se traducen en fuentes de beneficios. Es decir, que el capital social como fuente de regulación y cohesión intragrupales explica las formas en las que la estigmatización toma forma en las relaciones de los actores en el trabajo sexual.

En conclusión, desde la perspectiva del capital social de un trabajo no clásico, como el trabajo sexual, considero que los elementos conceptuales aquí esbozados pueden 
resultar útiles en el análisis de las relaciones sociales de mercados laborales que, como en el caso del comercio sexual, tienen dimensiones que desbordan la tradicional relación empleado-empleador y además permiten ampliar un planteamiento que parta desde las formas sociales que adquieren mercados laborales como el trabajo sexual para desentrañar mecanismos de exclusión y desigualdad social que han estado enraizados históricamente en esta actividad, sin que ello implique la imposibilidad de lógica de agencia y empoderamiento de los actores involucrados.

\section{Referencias}

Alcaldía Mayor de Bogotá, D. C. (9 de mayo de 2001). Decreto 400 de 2001. Por el cual se define la localización de las zonas de tolerancia y se reglamentan las condiciones para su funcionamiento. Registro Distrital 2387 del 9 de mayo de 2001. Recuperado de goo.gl/8aW74W

Araújo, N. (2009). A sociologia dos mercados de trabalho, ontem e hoje. Revista Novos estudos, 85, 151-170. DOI: 10.1590/S0101-33002009000300007

Berger, P. y Luckman, T. (1968). La construcción social de la realidad. Buenos Aires: Amorrortu Editores.

Carrasco, I., Castaño, M. S. y Pardo, I. (2011). Diferentes desarrollos del mercado de trabajo. ICE. Tendencias y Nuevos Desarrollos de la Teoría Económica, 858. Recuperado de goo.gl/6WTyvj

Corte Constitucional. (2010). Sentencia T/629 de 2010. M. P. J. C. Henao. Recuperado de goo.gl/URpXVe

De La Garza Toledo, E. (2010). Hacia un concepto ampliado de trabajo. Del concepto clásico al no clásico (Serie Cuadernos A, Temas de Innovación Social, 33). México: Anthropos/ UNAM-Iztapalapa.

Ding, Y. y Ho, P. S. Y. (2013). Sex work in China's pearl river delta: Accumulating sexual capital as a life-advancement strategy. Sexualities, 16(1-2), 43-60. Doi:10.1177/1363460712466211

Goffman, E. (1998). Estigma: la identidad deteriorada. Madrid: Amorrortu Editores.

Hakim, C. (2011). Erotic capital: The power of attraction in the bordroom and the bedroom. Basic Books: New York. 
Kalleberg, A. y Sorensen, A. B. (1979). The sociology of labor markets. Annual Review of Sociology, 5, 351-379.

Laverde, R. (2014). Impacto de la normatividad jurídica del trabajo sexual en la ciudad de Bogotá sobre las condiciones laborales y sociales de las mujeres trabajadoras sexuales (Tesis de posgrado). UNAM, México.

Maruani, M. (2000). De la sociología del trabajo a la sociología del empleo. Politica y Sociedad, 34, 9-17. Recuperado de goo.gl/dMh7F2

Murphy, A. y Ventakesh, S. (2011). Las carreras del vicio: los cambiantes contornos del trabajo sexual en la ciudad de Nueva York. En J. Auyaro y R. Hobert (Comp.), Acción e interpretación en la sociología cualitativa norteamericana (pp. 149-206). Quito: FLACSO Ediciones EPG.

Parker, R. y Aggleton, P. (2002). Estigma y discriminación relacionados con el VIH/Sida: un marco conceptual e implicaciones para la acción (Documentos de Trabajo n.o 9, Programa de Salud Reproductiva y Sociedad). México, D. F.: El Colegio de México.

Peck, J. (1996). Work-Place. The social regulation of labor markets. Nueva York: The Guilford Press.

Portes, A. (1999). Capital social: sus orígenes y aplicaciones en la sociología moderna. En J. Carpio e I. Novacovsky (Comp.), De igual a igual. El desafio del Estado ante los nuevos problemas sociales. México: Fondo de Cultura Económica - FLACSO.

Rivers-Moore, M. (2010). But the kids are okay: Motherhood, consumption and sex work in neo-liberal Latin America. The British Journal of Sociology, 61(4), 716-736. DOI: $10.1111 / \mathrm{j} .1468-4446.2010 .01338 . x$

Secretaría Distrital de Integración Social. (2009). Foro: Hablemos de prostitución. Bogotá: Autor.

Téllez, A. (2001). Trabajo y representaciones ideológicas de género. Propuesta para un posicionamiento analítico desde la antropología cultural. Gazeta de Antropología, 17. Recuperado de goo.gl/BJ5SkE

Shih, M. (2004). Positive stigma: Examining resilience and empowerment in overcoming stigma. Annals of the American Academy of Political and Social Science, 591 (Positive Development: Realizing the Potential of Youth), 175-185. Recuperado de goo.gl/ NQZtzI

Tilly, C. y Tilly, C. (1998). Worlds of work. En Work under capitalism (pp. 21-36). Boulder, CO: Westview Press. 